\title{
The Goldstone theorem and the Jahn-Teller effect
}

\author{
J. SARFATT $†$ and A. M. STONEHAM $\ddagger$ \\ $\dagger$ Department of Physics, University of Calıfornia, La Jolla, U.S.A. \\ ¥ Atomic Energy Research Establishment, Harwell, Didcot, Berks. \\ MS. recelved 25th November 1966
}

\begin{abstract}
The Goldstone theorem requires that a many-body system with broken symmetry has an excitation branch, whose irequency tends to zero in the limit of infinite wavelength. We treat a system where the broken symmetry comes from the terms which give rise to the Jahn-Teller effect. Both the excitation branches we discuss in detal have finite frequencies at infinite wavelength when there is no Jahn-Teller term; the introduction of this term forces one branch to have zerofrequency at infinite wavelength, in agreement with the Goldstone theorem The main point of this paper is this striking illustration of Goldstone's conjecture. Some of the simpler features of the excitation branches are discussed, they do not appear to have been treated in detail in the literature. Systems of ions in twofold degenerate E ground states may exhibit such excitations, which will have a characteristic velocity considerably less than that of sound.
\end{abstract}

\section{Introduction}

The non-relativistic form of the Goldstone theorem (Lange 1966) asserts that for a many-body system with 'broken symmetry' and with unscreened interactions of finite range there will be an excitation branch, whose frequency tends to zero in the limit of infinite wavelength. The term 'broken symmetry' means that the ground state is of infinite degeneracy, having lower symmetry than the Lagrangian, and that there is a continuous group of transformations which leaves the Lagrangian invariant but not the ground state. One of the better known examples of broken symmetry is given by the Heisenberg ferromagnet (Lange 1966). The ground state has infinite degeneracy, corresponding to the possible directions of magnetization, and has lower symmetry than the Lagrangian. The continuous symmetry group which changes the ground state and leaves the Lagrangian invariant is the rotation group generated by the components of the total spin, summed over all sites; the zero-frequency excitations are spin waves.

One general proof of this invokes Noether's theorem (Goldstone et al. 1962). The infinitesimal transformations of the continuous symmetry group are generated by the operator $\tilde{T}$ :

$$
\delta \phi_{\imath}=\delta\left(T_{\imath}, \phi_{3}\right)=\phi_{\imath}{ }^{\prime}-\phi_{\imath}
$$

where $\phi_{2}$ are the field variables describing the state of the system. The Lagrangian is invariant under $\tilde{T}$. The condition of broken symmetry may be expressed by the expectation value of the field variables in the exact ground state $|0\rangle$ being altered by the transformation which leaves the Lagrangian $\mathscr{L}$ invariant:

$$
\tilde{T}_{\imath \jmath}\left\langle 0\left|\phi_{,}\right| 0\right\rangle \neq 0 \text {. }
$$

Noether's theorem states that, given (1.1), there will exist a set of conserved currents:

conserved in the sense that

$$
J^{\mu}=i \frac{\partial \mathscr{L}}{\partial\left(\partial \phi_{l} / \partial X^{\mu}\right)} \tilde{T}_{\imath}, \phi_{1}
$$

$$
\partial_{\mu} J^{\mu}=0
$$

There will also be a conserved 'total charge operator'

$$
Q=\int d^{3} x J^{t}(x)
$$


which generates the continuous group of transformations

$$
\left[Q, \phi_{l}\right]=\tilde{T}_{i j} \phi_{j} .
$$

In $\S 3$ we will show directly that Noether's theorem applies to the specific system discussed.

The Jahn-Teller theorem (Jahn and Teller 1937) states that a system, such as a complex in a solid or a molecule, which has a ground state possessing any degeneracy other than the Kramers degeneracy, can achieve a lower energy by a distortion which removes the degeneracy. The ground state then has lower symmetry than the Lagrangian. In some cases there are an infinite number of possible distortions which lower the energy equally. The continuous group of transformations transforms the ground state obtained by one such distortion into that from a slightly different distortion. For example we shall discuss the tetragonal distortions of octahedral complexes. The continuous group changes a tetragonal distortion along one cubic axis of the complex into one with small additional distortions along the other axes. This would alter the ground state without changing the Lagrangian.

In $\$ 2$ we discuss an array of $\mathrm{Cu}^{2+}$ ions specifically and calculate the Lagrange density in a continuum approximation. The $\mathrm{Cu}^{2+}$ complex has been discussed many times before (for general reference we cite Stoneham 1965 and Goodenough 1963, p. 202 et seq.). The Lagrange density is used to obtain the classical ground state and equations of motion in $\S 3$. In this section we will also verify Noether's theorem explicitly for this system. In the ground state all the complexes in the array have the same distortion configuration. The equations of motion are greatly affected by the presence of the terms which give rise to the Jahn-Teller effect. When these terms are absent the excitations have finite frequency at infinite wavelength. If the Jahn-Teller terms are present, one excitation branch has zero frequency at infinite wavelength. This change on the introduction of the symmetry breaking terms is in exact agreement with the predictions of the Goldstone theorem, and the striking illustration of the theorem is the main point of this paper. We discuss the excitations themselves in $\$ 4$. Here we also consider briefly the effect of additional terms in the Lagrangian which give a physically more realistic model. These terms destroy the broken symmetry, but do not necessarily remove the zero frequency at infinite wavelength; we are thus able to demonstrate that the Goldstone theorem is sufficient but not necessary for such a branch.

\section{The Lagrangian density for an array of Jahn-Teller ions}

As an explicit example we consider $\mathrm{Cu}^{2+}$ ions in an otherwise non-magnetic host lattice. We further assume that the magnetic ions have six nearest neighbours which would be arranged octahedrally in the absence of a Jahn-Teller effect.

In the undistorted cubic environment the ground state of the $\mathrm{Cu}^{2+}$ ion is orbitally degenerate. The two degenerate states, $|\epsilon\rangle$ and $|\theta\rangle$, transform as $x^{2}-y^{2}$ and $2 z^{2}-x^{2}-y^{2}$ respectively under the octahedral group $\mathrm{O}_{\mathrm{h}}$ and they form a basis for an E-type representation of the group. We suppress the spin variables in our notation as the spin-orbit coupling has no elements among $|\epsilon\rangle$ and $|\theta\rangle$ and because the Kramers degeneracy is not important in the present context.

A ground state with lower energy may be achieved by a tetragonal distortion of the complex of the $\mathrm{Cu}^{2+}$ ion and its nearest neighbours. Of the three possible tetragonal distortions (the distortions along the three distinct cubic axes) only two are independent. We make the standard choice (Van Vleck 1939) of $\epsilon, \theta$, where

$$
\begin{aligned}
& X_{1}=-X_{4}=+\frac{\epsilon}{2}-\frac{\theta}{2 \sqrt{ } 3} \\
& Y_{2}=-Y_{5}=-\frac{\epsilon}{2}-\frac{\theta}{2 \sqrt{ } 3} \\
& Z_{3}=-Z_{6}=+\frac{\theta}{\sqrt{ } 3}
\end{aligned}
$$


in which $X_{1}$ is the $x$ displacement of neighbour 1 in the complex, and so on. The labelling of the atoms and $\epsilon$ and $\theta$ are illustrated in figure 1 . The normal coordinates of the complex, $\epsilon$ and $\theta$, which are distortions, should not be confused with the electronic wave functions $|\epsilon\rangle$ and $|\theta\rangle$. The similarity of notation is standard and occurs because, for example, $\epsilon$ and $|\epsilon\rangle$ transform in the same way under the octahedral group. In addition to $\epsilon$ and $\theta$ there are, of course, other possible distortions of the complex. We shall ignore these as they introduce no essential changes. We also ignore all effects of surfaces.

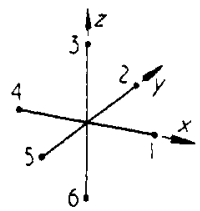

(a)

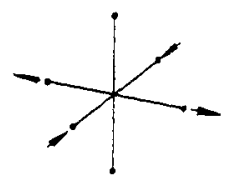

(b)

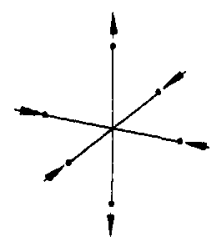

(c)

Figure 1. (a) The labelling of the nearest neighbours to a $\mathrm{Cu}^{2}+10$, (b) the $\epsilon$ distortion, (c) the $\theta$ distortion.

In general $\epsilon$ and $\theta$ will be different for the different complexes in the array. We shall use a continuum approximation and write $\epsilon(x)$ and $\theta(x)$, where $x$ is the coordinate of the complex. $\epsilon$ and $\theta$ will be treated as continuous functions of $x$, and our discussion will be in terms of energy densities rather than energies. The continuum approximation allows us to simplify the notation and to introduce phenomenological coefficients without having to make sophisticated assumptions about the microscopic features of the system. Strictly speaking $\epsilon(x)$ and $\theta(x)$ are averages over a region large enough to contain many complexes, but sufficiently small for $\epsilon$ and $\theta$ to be essentially constant in the region. Clearly such a picture is only useful when $\epsilon$ and $\theta$ vary slowly in space, that is for long wavelength excitations.

In our simple model there are three contributions to the potential energy density. First, a contribution quadratic in the distortions comes from the usual forces which maintain the crystal equilibrium:

$$
V_{\mathrm{a}}=A\left(\epsilon^{2}+\theta^{2}\right) \text {. }
$$

Secondly, when $\epsilon$ and $\theta$ vary in space there will be a term

$$
V_{b}=\frac{1}{2} B\left\{\left(\frac{\partial \epsilon}{\partial X}\right)^{2}+\left(\frac{\partial \theta}{\partial X}\right)^{2}\right\}
$$

in which $B$ is an effective tension representing the interaction between the complexes. Both $V_{\mathrm{a}}$ and $V_{\mathrm{b}}$ have the same expectation value for $|\epsilon\rangle$ as they do for $|\theta\rangle$. Our particular choice of $\epsilon$ and $\theta$ ensures that, from symmetry arguments alone, we know that $\epsilon$ and $\theta$ enter symmetrically in $V_{\mathrm{a}}$ and $V_{\mathrm{b}}$ and that there are no cross terms involving $\epsilon \theta$ or $(\partial \epsilon / \partial X)(\partial \theta / \partial X)$. The final contribution is the one which is responsible for the Jahn-Teller distortion. The matrix elements among $|\epsilon\rangle,|\theta\rangle$ which correspond to distortions $\epsilon$ and $\theta$ are

$$
\begin{array}{c|rr} 
& |\theta\rangle & |\epsilon\rangle \\
\langle\theta| & -C \theta & C \epsilon \\
\langle\epsilon| & C \epsilon & +C \theta \\
\hline
\end{array}
$$

These matrix elements are the only contributions to the matrix of the Hamiltonian, $H_{\imath,}$, which are not proportional to the unit matrix. All the other terms $\left(V_{\mathrm{a}}, V_{\mathrm{b}}\right.$, the electronic energy for zero distortion and the kinetic energy) have the same expectation value for $|\epsilon\rangle$ and $|\theta\rangle$. The solution of the secular equation $\operatorname{det}\left|H_{1}-E \delta_{v}\right|=0$ to find the energy for given $\epsilon$ and $\theta$ is therefore trivial. It is readily shown that the effect of the terms (2.4) is to lower one eigenvalue by $C\left(\epsilon^{2}+\theta^{2}\right)^{1 / 2}$ and to raise the other by the same amount. For 
the ground state we write accordingly

$$
V_{\mathrm{c}}=-C\left(\epsilon^{2}+\theta^{2}\right)^{1 / 2}
$$

In addition to the potential energy terms there is a kinetic energy density

$$
T=\frac{1}{2} \rho\left\{\left(\frac{\partial \epsilon}{\partial t}\right)^{2}+\left(\frac{\partial \theta}{\partial t}\right)^{2}\right\}
$$

where $\rho$ is the effective density of the complexes. The Lagrangian density is thus

$$
\begin{aligned}
\mathscr{L} & =T-\left(V_{\mathrm{a}}+V_{\mathrm{b}}+V_{\mathrm{c}}\right) \\
& =\frac{1}{2} \rho\left\{\left(\frac{\partial \epsilon}{\partial t}\right)^{2}+\left(\frac{\partial \theta}{\partial t}\right)^{2}\right\}-A\left(\epsilon^{2}+\theta^{2}\right)-\frac{1}{2} B\left\{\left(\frac{\partial \epsilon}{\partial X}\right)^{2}+\left(\frac{\partial \theta}{\partial X}\right)^{2}\right\}+C\left(\epsilon^{2}+\theta^{2}\right)^{1 / 2} .
\end{aligned}
$$

This may be written more elegantly by introducing a field variable $\phi(x)=\epsilon(x)+i \theta(x)$ such that $\phi^{*} \phi=\epsilon^{2}+\theta^{2}$. The Lagrange density becomes

$$
\mathscr{L}=\frac{1}{2} \rho \frac{\partial \phi^{*}}{\partial t} \frac{\partial \phi}{\partial t}-A \phi^{*} \phi-\frac{1}{2} B \frac{\partial \phi^{*}}{\partial x} \frac{\partial \phi}{\partial x}+C\left(\phi^{*} \phi\right)^{1 / 2} .
$$

The important features to note are first that the Lagrangian density $\mathscr{L}$ still has full cubic symmetry, whereas the ground state has lower (tetragonal) symmetry. Secondly, $\mathscr{L}$ is invariant under the continuous internal symmetry transformation

$$
\begin{aligned}
\theta(x) \rightarrow \theta^{\prime \prime}(x) & =\theta(x) \cos \alpha+\epsilon(x) \sin \alpha \\
\epsilon(x) \rightarrow \epsilon^{\prime \prime}(x) & =-\theta(x) \sin \alpha+\epsilon(x) \cos \alpha
\end{aligned}
$$

where $\sin ^{2} \alpha+\cos ^{2} \alpha=1$. This transformation does not, however, leave the Jahn-Teller ground state invariant; it changes a tetragonal distortion entirely along one of the cubic axes into a distortion with admixtures of tetragonal distortions along the other axes. The Jahn-Teller ground state has an infinite degeneracy corresponding to the choice of the phase $\alpha$. This symmetry, described by (2.9), is the 'broken symmetry' of the Goldstone theorem; it is an internal symmetry as these transformations leave the Lagrangian invariant, but is broken in the sense that the ground state is not invariant. The reduction of cubic symmetry to tetragonal symmetry by the Jahn-Teller effect is not the broken symmetry of the Goldstone theorem.

\section{The classical ground state and the Lagrangian equations of motion}

In this section we find the ground state of the system and, from the equations of motion, the low-lying excitations of the system. The classical approach we adopt has several features which are simpler than in a quantum-theoretic approach. The ground state is almost obvious inturtively and there are no complications of zero point motion. Further, one may consider oscillations about equilibrium of indefinitely small amplitude and one may ignore all questions of the limitations of possible experiments by the uncertainty principle. The Goldstone conjecture appears to be equally valid in the classical and quantal cases.

The classical ground state will be a static state with no kinetic energy. The only term in the potential energy which depends on the spatial variation of and $\theta$ is

$$
\frac{1}{2} B\left(\partial \phi^{*} / \partial x\right)(\partial \phi / \partial x)
$$

where $B$ is positive. Clearly $\epsilon$ and $\theta$ are constants in space in the classical ground state, thereby minimizing this term. Consequently the ratio $\epsilon(x) / \theta(x) \equiv \tan \{\psi(x)\}$ and the magnitude of $\phi^{*}(x) \phi(x)=\epsilon^{2}(x)+\theta^{2}(x)$ are independent of $x$. The phase $\psi(x)$ does not enter into the other terms in the potential energy, so, without loss of generality, we may take $\psi(x)=0$ in the ground state. With this phase convention the ground-state distortion configuration is

$$
\{\epsilon(x), \theta(x)\}_{0} \equiv\left(0, \theta_{0}\right) .
$$


By straightforward minimization of the terms in the energy which depend on $\theta_{0}$ we find the magnitude of $\theta_{0}$ to be

$$
\theta_{0}=\left\{\left(\epsilon^{2}+\theta^{2}\right)^{1 / 2}\right\}_{0}=\frac{C}{2 A} .
$$

The Lagrangian equations of motion are given by

$$
\frac{\partial \mathscr{L}}{\partial \phi}=\frac{\partial}{\partial t} \frac{\partial \mathscr{L}}{\partial(\partial \phi / \partial t)}+\frac{\partial}{\partial x} \frac{\partial \mathscr{L}}{\partial(\partial \phi / \partial x)} .
$$

Explicit evaluation of these gives

$$
\begin{aligned}
& \rho \frac{\partial^{2} \epsilon}{\partial t^{2}}=C \frac{\epsilon}{\left(\epsilon^{2}+\theta^{2}\right)^{1 / 2}}-2 A \epsilon+B \frac{\partial^{2} \epsilon}{\partial x^{2}} \\
& \rho \frac{\partial^{2} \theta}{\partial t^{2}}=C \frac{\theta}{\left(\epsilon^{2}+\theta^{2}\right)^{1 / 2}}-2 A \theta+B \frac{\partial^{2} \theta}{\partial x^{2}} .
\end{aligned}
$$

For future reference we observe that $\epsilon /\left(\epsilon^{2}+\theta^{2}\right)^{1 / 2}$ and $\theta /\left(\epsilon^{2}+\theta^{2}\right)^{1 / 2}$ are finite for all finite values of $\epsilon$ and $\theta$ including $\epsilon=\theta=0$. Thus in cases where $C$ is zero (no Jahn-Teller effect) the terms in $C$ simply vanish.

We now consider small oscillations about the ground-state configuration $(\epsilon=0$, $\left.\theta=\theta_{0}\right)$. The equations of motion are more transparent after the transformation:

$$
\theta \rightarrow \theta^{\prime}=\theta-\theta_{0}, \quad \epsilon \rightarrow \epsilon
$$

where $\epsilon$ and $\theta^{\prime}$ are small for small oscillation amplitudes. We also linearize the equations, assuming $\theta_{0} \gg \epsilon, \theta^{\prime}$; the terms omitted are anharmonic terms of the order of $\epsilon^{2}, \theta^{\prime 2}, \epsilon \theta^{\prime}$. Finally, we find for $\theta^{\prime}$

$$
\rho \frac{\partial^{2} \theta^{\prime}}{\partial t^{2}}=B \frac{\partial^{2} \theta^{\prime}}{\partial x^{2}}-2 A \theta^{\prime}+\mathrm{O}\left(\theta^{\prime} \epsilon, \theta^{\prime 2}\right)
$$

which should be compared with the original equation of motion in the absence of the Jahn-Teller term, i.e.

$$
\rho \frac{\partial^{2} \theta}{\partial t^{2}}=B \frac{\partial^{2} \theta}{\partial x^{2}}-2 A \theta \text {. }
$$

These two equations of motion are identical, apart from the anharmonic correctionsthe $\theta^{\prime}$ oscillations are not affected by the Jahn-Teller term. On the other hand, for $\epsilon$

$$
\rho \frac{\partial^{2} \epsilon}{\partial t^{2}}=B \frac{\partial^{2} \epsilon}{\partial x^{2}}+\mathrm{O}\left(\epsilon^{2}, \theta^{\prime} \epsilon\right)
$$

including the Jahn-Teller term. Here the oscillations in $\epsilon$ are now acoustic in nature, having a frequency which tends to zero as the wavelength becomes infinite. This is in complete contrast with the corresponding equation of motion with no Jahn-Teller term, when the excitations have a finite frequency even at infinite wavelength:

$$
\rho \frac{\partial^{2} \epsilon}{\partial t^{2}}=B \frac{\partial^{2} \epsilon}{\partial x^{2}}-2 A \epsilon
$$

The acoustic-like excitation described by (3.9) is quite distinct from the usual acoustic phonon branch. The broken symmetry due to the Jahn-Teller effect forces a zero frequency excitation of infinite wavelength to come into existence in conformity with Goldstone's theorem.

We may also verify that Noether's theorem applies to our system. From (2.9) we see that for an infinitesimal rotation ( $\alpha$ small)

$$
\begin{aligned}
& \theta \rightarrow \theta^{\prime \prime}=\theta+\alpha \epsilon \\
& \epsilon \rightarrow \epsilon^{\prime \prime}=\epsilon-\alpha \theta
\end{aligned}
$$


i.e.

$$
\begin{aligned}
& \delta \theta \equiv \theta^{\prime \prime}-\theta=\alpha \epsilon \\
& \delta \epsilon \equiv \epsilon^{\prime \prime}-\epsilon=-\alpha \theta .
\end{aligned}
$$

The matrix $\tilde{T}_{2}$ is, writing $\theta=\phi_{1}$ and $\epsilon=\phi_{2}$,

$$
\tilde{T}_{\eta}=\left(\begin{array}{rr}
0 & 1 \\
-1 & 0
\end{array}\right)
$$

Equation (1.3), which defines the currents, and (2.8), which gives the Lagrangian, lead to current components

$$
\begin{aligned}
& J^{x}=-B\left(\frac{\partial \theta}{\partial x} \epsilon-\frac{\partial \epsilon}{\partial x} \theta\right) \\
& J^{t}=+\rho\left(\frac{\partial \theta}{\partial t} \epsilon-\frac{\partial \epsilon}{\partial t} \theta\right) .
\end{aligned}
$$

It is then easy to verify the current conservation condition of Noether's theorem:

$$
\begin{aligned}
\partial_{\mu} J^{\mu}=\frac{\partial J^{x}}{\partial x}+\frac{\partial J^{t}}{\partial t} & =\epsilon\left(\rho \frac{\partial^{2} \theta}{\partial t^{2}}-B \frac{\partial^{2} \theta}{\partial x^{2}}\right)-\theta\left(\rho \frac{\partial^{2} \epsilon}{\partial t^{2}}-B \frac{\partial^{2} \epsilon}{\partial x^{2}}\right) \\
& =-\epsilon\left(\frac{C}{\left(\epsilon^{2}+\theta^{2}\right)^{1 / 2}}-2 A\right) \theta+\theta\left(\frac{C}{\left(\epsilon^{2}+\theta^{2}\right)^{1 / 2}}-2 A\right) \epsilon=0
\end{aligned}
$$

where the equations of motion (3.4) and (3.5) have been used in the final step. The conserved charge $Q$, defined by $(1.5)$, is

$$
Q=\int d^{3} x J^{t}(x)=\rho \int d^{3} x\left(\epsilon \frac{\partial \theta}{\partial t}-\theta \frac{\partial \epsilon}{\partial t}\right)=-\rho \int d^{3} x\left(\epsilon^{2}+\theta^{2}\right) \frac{\partial \psi}{\partial t}
$$

where $\psi=\tan ^{-1}(\epsilon / \theta)$. In principle this constant of the motion could be obtained by direct integration of (3.8) and (3.10). $Q$ is essentially the total angular momentum associated with rotations in the $(\epsilon, \theta)$ space.

\section{Discussion of the excitations in $\epsilon$ and $\theta^{\prime}$}

Here we attempt to clarify the nature of the $\epsilon$ and $\theta^{\prime}$ waves further. As mentioned in $\S 2, \epsilon$ and $\theta^{\prime}$ are independent types of distortion which are orthogonal in the sense that they provide different components of a basis for an irreducible representation of the octahedral group. We can consider the excitations as oscillations in the configurations of the complexes in a configuration space with coordinates $\epsilon$ and $\theta$. This configuration space 1s 1 llustrated in figure 2 which also shows the potential energy as a function of $\epsilon$ and $\theta$. In the ground state all the complexes have the same configuration $\left(0, \theta_{0}\right)$. This is, of course, analogous to the ferromagnetic ground state where the spins on each site are in the same state.

The continuous group of transformations which change the ground state but not the Lagrangian are given in $(2.9)$ : these transformations are simply rotations in $(\epsilon, \theta)$ space. The $\theta^{\prime}$ excitations are oscillations in the magnitude of $\theta$, the symmetry breaking coordinate. Their finite frequency at infinite wavelength occurs because motion in $\theta^{\prime}$ takes each complex up the sides of the potential trough shown in figure 2 . On the other hand, the motion in $\epsilon$, which is orthogonal to $\theta^{\prime}$, simply moves the complex along the minimum of the trough; in the limit of infinite wavelength these excitations have zero frequency. The velocity of propagation of these excitations is determined by the coefficient $B$ of $(2.3)$ which is a measure of the interaction between complexes tending to give them the same $(\epsilon, \theta)$. Clearly this may be very small in dilute systems. In other systems the interaction may be appreciable, as in the spinel $\mathrm{Mn}^{2+}\left(\mathrm{Mn}^{3+}\right)_{2} \mathrm{O}_{4}$, which is magnetically dense and which exhibits a cooperative Jahn-Teller distortion below $1443^{\circ} \mathrm{K}$ where it exhibits a diffusionless 
phase transition (Goodenough 1963, p. 226). A very crude estimate of the propagation velocity, $(B / \rho)^{1 / 2}$, can be made as follows. If there are $N$ complexes per unit volume, each of effective mass $m$, the density $\rho$ is $N m$ The critical temperature of $1443{ }^{\circ} \mathrm{K}$ suggests an interaction between neighbouring complexes of the order of $0.1 \mathrm{ev}$. The energy density $B$,

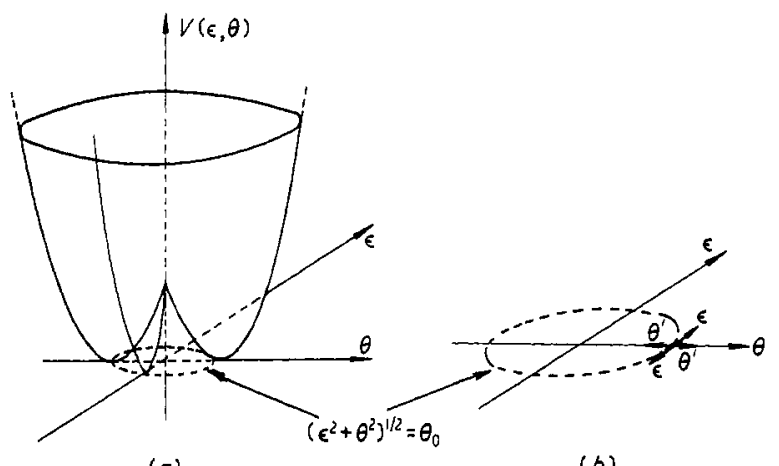

(a)

(b)

Figure 2. (a) The potential energy as a function of $\epsilon$ and $\theta$; $(b)$ the oscillations in $\epsilon$ and $\theta^{\prime}$.

may then be of order $\frac{1}{2} Z N 0.1 \mathrm{ev}$, where $Z$ is the number of complexes which are nearest neighbours to a given complex, and the $\frac{1}{2}$ ensures that the pairwise interactions are only counted once. Using $Z=12$ and the atomic weight of oxygen for $m$ we find a propagation velocity of $2 \times 10^{3} \mathrm{~cm} \mathrm{sec}^{-1}$, appreciably less than the velocity of sound.

The configuration of our system may change without a change in energy by altering $\psi=\tan ^{-1}(\epsilon \mid \theta)$ with $\epsilon^{2}+\theta^{2}=\theta_{0}{ }^{2}$. This means that for our system the Jahn-Teller effect would be dynamic even at the lowest temperatures. In general the potential energy may depend on $\psi$ through a term $V_{\mathrm{d}}(\psi)$; for a static Jahn-Teller effect $V_{\mathrm{a}}$ leads to discrete minima in $V(\theta, \epsilon)$ separated by potential barrers high enough to make tunnelling improbable. The broken symmetry necessary for the Goldstone theorem is destroyed by $V_{\mathrm{d}}(\psi)$-the Lagrangian is no longer invariant under (2.9)-although the excitation branch at infinite wavelength is not necessarily affected. The Goldstone theorem is sufficient but not necessary for the existence of such a branch. This can be seen for our system as follows. Let us suppose the new anisotropic terms $V_{\mathrm{a}}(\psi)$ lead to the lowest energy at $\psi=\psi_{0}$. Near $\psi=\psi_{0}$ the potential energy along $\theta^{2}+\epsilon^{2}=\theta_{0}^{2}$ will have the form

$$
V_{\mathrm{d}}(\psi)=D\left(\psi-\psi_{0}\right)^{2}+\text { higher-order terms. }
$$

$D$ and the higher-order terms depend on the specific model considered. If $D$ is zero, the higher terms alone simply give anharmonic corrections to (3.9); these terms destroy the broken symmetry on which Goldstone's theorem depends, but leave the excitation spectrum unaltered. In general $D$ is finite for real systems, although it may be very small-for $\mathrm{MgO}: \mathrm{Cu}^{2+}$ the Jahn-Teller effect remains dynamic down to liquid helium temperatures (Orton et al. 1961), so the discrete minima in $V$ are separated by barriers of order $0.0005 \mathrm{ev}$. For finite $D$ the dispersion relation regains an energy gap.

\section{Conclusion}

The Jahn-Teller terms in the Lagrangian of the system we have treated produce a system with broken symmetry. We have examined the excitations of this system and find that the inclusion of these terms forces one excitation branch to have zero frequency at infinite wavelength, giving a very clear illustration of Goldstone's conjecture. The resulting excitations have been discussed briefly; in real systems they will usually have a propagation velocity less than that of sound, and may have an energy gap, which will be small in some cases. There appears to be no detailed discussion of these excitations in the literature. 


\section{Acknowledgments}

We wish to thank Dr. J. Beeby for helpful discussions and Dr. P. W. Anderson for valuable comments. One of us (J.S.) would like to thank the Atomic Energy Research Establishment, Harwell, for a vacation associateship during the tenure of which this work was performed, and also to acknowledge a travel grant from the U.S. National Science Foundation.

\section{References}

Goldstone, J., Salam, A., and Weinberg, S., 1962, Phys. Rev., 127, 965-70.

Goodenough, J. B., 1963, Magnetism and the Chemical Bond (New York. John W1ley).

Jahn, H. A., and Telier, E., 1937, Proc. Roy. Soc. A, 161, 220-35.

Lange, R. V., 1966, Phys. Rev., 146, 301-3.

Orton, J. W., Auzins, P., Griffiths, J. H. E., and Wertz, J. E., 1961, Proc. Phys. Soc., 78, 554-68.

Stoneham, A. M., 1965, Proc. Phys. Soc., 85, 107-17.

Van Vleck, J. H., 1939, J. Chem. Phy's., 7, 72-84. 